Revue

d'ethnoécologie
Revue d'ethnoécologie

13 | 2018

Varia + Dossier "Anthropologie maritime"

\title{
Les « terroirs » maritimes revisités
}

Fin ou renouveau dans le contexte de la globalisation des mers et des littoraux ouest-africains?

Revisiting the marine commons or "terroirs". End or revival in the context of the sea globalisation and in the West-African coast governance?

\section{Marie-Christine Cormier-Salem et Adama Mbaye}

\section{(2) OpenEdition}

\section{Journals}

Édition électronique

URL : http://journals.openedition.org/ethnoecologie/3433

DOI : $10.4000 /$ ethnoecologie.3433

ISSN : 2267-2419

Éditeur

Laboratoire Eco-anthropologie et Ethnobiologie

Référence électronique

Marie-Christine Cormier-Salem et Adama Mbaye, «Les « terroirs » maritimes revisités », Revue d'ethnoécologie [En ligne], 13 | 2018, mis en ligne le 25 juin 2018, consulté le 19 avril 2019. URL : http:// journals.openedition.org/ethnoecologie/3433 ; DOI : 10.4000/ethnoecologie.3433

Ce document a été généré automatiquement le 19 avril 2019

Revue d'ethnoécologie est mis à disposition selon les termes de la licence Creative Commons Attribution - Pas d'Utilisation Commerciale - Pas de Modification 4.0 International. 


\section{Les « terroirs » maritimes revisités}

Fin ou renouveau dans le contexte de la globalisation des mers et des littoraux ouest-africains?

Revisiting the marine commons or "terroirs». End or revival in the context of

the sea globalisation and in the West-African coast governance?

Marie-Christine Cormier-Salem et Adama Mbaye

\section{Introduction}

1 La diversité des formes de socialisation de l'espace maritime et de spatialisation des sociétés maritimes, selon l'heureuse définition de la géographie par Sautter (1973), se traduit dans la richesse des termes pour désigner les espaces structurés par l'exploitation de la mer et de ses ressources et les droits traditionnels d'appropriation, d'usage et d'accès qui y sont attachés, ou les tenures maritimes, des TUR'f (Territorial Use Rights in fisheries) au CMT (Customary Marine Tenure), des terroirs maritimes aux technotopes (Cormier-Salem sous presse).

2 Cette contribution se propose de revisiter la notion de terroir maritime, en s'appuyant sur des études empiriques menées en particulier au Sénégal depuis les années 1980 (Cormier-Salem 1992, 2000) et en ré-explorant cette notion à partir de terrains diversifiés en Afrique de l'Ouest (Mbaye \& Cormier-Salem 2015) et à l'aune des discours et dispositifs environnementaux globaux récents.

De fait, depuis les années 1980s, la prise de conscience de la crise du secteur halieutique ou "surpêche " (Cury \& Cayré 2001, Pauly 1998), dans un contexte de changement environnemental global, conduit à une remise en cause des modèles classiques de gestion des pêches et à de nouvelles politiques publiques ${ }^{1}$ (Cormier-Salem sous presse). Ces politiques sont le plus souvent édictées au niveau international, déclinées à l'échelle des Etats, mises en œuvre à l'échelle locale, comme c'est le cas en Afrique de l'Ouest. La globalisation des discours, des politiques et des mécanismes (juridiques, financiers, économiques) dans le champ de l'environnement, et la montée en puissance du néolibéralisme ont fait l'objet de nombreux travaux critiques $^{2}$. Dans cette contribution, 
nous nous intéressons aux effets de la globalisation de la mer sur la gouvernance locale des littoraux sénégalais. Ainsi, face à l'injonction internationale à mettre en place des Aires Marines Protégées (AMP) et à y faire participer les communautés locales, nous nous interrogeons sur l'accaparement public et privé du littoral et analysons la diversité des formes de résistances et arrangements institutionnels de la part des pêcheurs artisans, en donnant un éclairage sur les anciens et nouveaux terroirs maritimes.

Notre cadre théorique, conceptuel et méthodologique s'inspire tout à la fois de la géographie (et en particulier de l'école africaniste des terroirs), de l'anthropologie maritime et de Political ecology (Zimmerer 2006). Tout en portant un regard critique sur les discours dominants (globalism, environmentalism, néocolonialisme, néoliberalisme) (Perreault et al. 2015), nous privilégions l'analyse des interactions entre acteurs, normes, institutions, nous efforçant de mettre en évidence les diverses gouvernementalités à propos de l'environnement maritime (y compris littoral ${ }^{3}$ ). Nous nous intéressons particulièrement aux mécanismes d'injustice environnementale ${ }^{4}$ et à la notion d'accaparement de la mer (ocean grabbing). En référence à l'accaparement des terres à des fins de production land grabbing (Saturnino et al. 2011) ou de conservation green grabbing (Fairhead et al. 2013), l'accaparement de la mer, ocean grabbing, désigne la réallocation de ressources et espaces maritimes suite à des projets, publics ou privés, de conservation, aménagement ou développement, qui induisent une gouvernance préjudiciable aux communautés, celles-là même dont l'existence dépend de ces ressources et espaces (Bennett et al. 2015). Les créations d'aires protégées, en changeant les régimes de propriétés et d'accès à la mer et ses ressources, en dépossédant les pêcheurs artisans de leurs terroirs et territoires de pêche, comptent parmi les principaux mécanismes d'enclosure des communaux maritimes.

Notre méthodologie combine approches théoriques et empiriques. Nos travaux de terrain, tout en privilégiant le niveau local (cartographie participative des terroirs maritimes, recueil des savoirs locaux), s'efforcent de développer une démarche multi scalaire, intégrée, et diachronique. Nos données sont issues d'enquêtes qualitatives sur la longue durée (années 1980-2016 pour Cormier-Salem, 1997-2016 pour Mbaye), mais aussi quantitatives à travers, par exemple, la participation au recensement annuel du parc piroguier du Sénégal effectué par le Centre de Recherche Océanographique de Thiaroye (CRODT). Elles sont aussi issues de recherches documentaires sur les interventions publiques, les textes réglementaires, les dispositifs nationaux et internationaux. Elles se nourrissent de travaux scientifiques, anciens comme ceux conduits par l'Orstom, devenu IRD, publiés dans la collection de l'atlas des terroirs et structures agraires jusqu'à ceux plus récents du programme « Retour sur des terroirs anciens » (Blanc-Pamard \& Boutrais 1997), mais aussi de la littérature internationale (Zimmerer \& Bassett 2003, Zimmerer 2006).

6 Dans cette contribution, nous discutons de la pertinence de la notion de terroir maritime et proposons un cadre conceptuel et méthodologique. Nous retraçons la chronique de la fin de ces terroirs ou de leur remise en cause avec les processus d'enclosure ${ }^{5}$; puis nous analysons leur renouveau, compte tenu du changement de paradigme en termes de conservation de la biodiversité et des nouvelles approches de cogestion, se focalisant sur le local. Cette dynamique est discutée à travers divers exemples ouest-africains, de l'échelle internationale au local: le projet de territorialisation des espaces maritimes ouest-africains de la Banque Mondiale, l'acte III de la décentralisation, achevant le processus de communalisation des terroirs traditionnels et la création des aires ou zones 
marines protégées au Sénégal, dites communautaires ou patrimoniales telles les APAC (Aires du Patrimoine Autonomes et Communautaires) et les ZPP (Zones de Pêche Prioritaire), ou encore l'engouement actuel pour les produits de terroir ou de meroir (Mbaye et al. 2018, Cormier-Salem 2015, Sarr et al. 2009).

\section{La notion de terroir maritime}

7 La notion de terroir maritime est inspirée de diverses perspectives, en géographie, écologie politique et anthropologie sociale, qui ont pour points communs de considérer les interactions sociétés-natures dans la longue durée et de les analyser en privilégiant les catégories locales et les savoirs, pratiques et stratégies des communautés. La notion de terroir est typiquement francophone, voire africaniste, à l'origine d'une école (Sautter \& Pélissier 1964, Pélissier \& Sautter 1970), fortement critiquée par certains auteurs (Brunet et al. 1992), nuancée, voire remise en cause par ceux-là même qui l'avaient promue (Pélissier 1995), réinvestie par d'autres, notamment des anthropologues (Fay 1989, Verdeaux 1992, Chauveau et al 2000, Lavigne-Delville 2001). Cette notion, utilisée à l'origine pour désigner les portions de territoire dont dépendent les communautés rurales pour leur subsistance, nous avait semblé pertinente pour qualifier les espaces contrôlés par les paysans-pêcheurs et que nous distinguions des parcours des marinspêcheurs (Cormier-Salem 1995a). Sans revenir sur le détail de notre argumentaire, rappelons que la notion d'espace géographique désigne, dialectiquement, un support physique (substrat, haut-fond..), un produit historique et social (paysage, socioécosystème..) et un enjeu de rapports sociaux (compétition, allocation..). Le territoire est une construction plus symbolique et politique de l'espace, un référent identitaire et patrimonial. La nature tridimensionnelle de la mer et la mobilité des ressources halieutiques rendent plus complexe l'appréhension, la délimitation et l'appropriation de ce ces espaces.

Contrairement à l'approche bioécologique classique, l'espace maritime n'est pas considéré comme une donnée naturelle stockant des populations de poissons représentant la ressource halieutique, mais comme une construction sociale (CormierSalem 2000). La gestion des ressources halieutiques ne peut être réduite à la gestion du support physique, mais doit prendre en compte les différentes dimensions et représentations de l'espace. Les espaces halieutiques sont définis en fonction des pratiques et des représentations propres à chaque groupe d'acteurs de chaque pêcherie mais aussi en fonction des savoirs locaux. Loin d'être de simples entités topographiques, physiquement bornées, les unités spatiales renvoient à des systèmes de pêche dont les éléments sont, selon les termes de Corlay (1979), biologiques (biomasse, ressources exploitées), techniques (moyens de production en mer et de traitement à terre), économiques (structures financières des armements, vocation et destination des captures, partenaires commerciaux), sociaux (producteurs et consommateurs impliqués), culturels (pratiques alimentaires et religieuses, perception de l'environnement etc.) et politiques (réglementations aides). Les espaces halieutiques désignent ainsi les entités spatiales structurées par les systèmes de pêche, à la fois support physique des activités halieutiques, produits des pratiques et représentations des sociétés littorales et enfin enjeux et, donc, source de conflits entre communautés pour le contrôle des ressources maritimes. 
9 Cette vision écosystémique des pêches se retrouve chez Revéret (1991) qui remarque qu'au-delà des zones de pêche délimitées par les organismes de gestion, il existe dans les pêcheries artisanales tout un système d'appellations toponymiques qui témoignent de la permanence des sites pour les pêcheurs. Ces derniers n'ont pas par conséquent la perception de pêcher un stock, unité de gestion du biologiste, mais bien de contrôler des lieux de pêche clairement identifiés. Par exemple à Saint-Louis, les pêcheurs citent souvent birago, ngor, marie diop, madaga, gajaga, kër saas, marem siru, tapha diãne, leydi gaye, jatara, hôpital, tolu tandarma, praya, etc. Les pêcheurs opérant dans des zones rocheuses fréquentées par les espèces démersales, donnent une nomenclature aux rochers appelés xeer en wolof à partir de repères terrestres. À ces lieux, sont attachées des techniques (ou engins de pêche), des pratiques, des institutions, désignées sous le terme de technotopes par Fay (1989).

10 Le statut des unités spatiales, telles que découpées dans l'activité de pêche, la façon dont elles sont exploitées, aménagées, perçues et partagées, autrement dit la façon dont elles sont gérées et gouvernées, est la question centrale en halieutique. Les espaces structurés par les pratiques halieutiques, liquides et mouvants, aux frontières poreuses, ne sont pas des res nullius, mais des res communes ou communaux, sous le contrôle d'une communauté qui s'identifie en eux, les revendique comme son territoire et en assure la défense et la conservation (Cormier-Salem 1995a, Cormier-Salem sous presse).

Dans les socio-écosystèmes semi-clos (lagunes, estuaires, mangrove) ou insulaires (récifs coralliens, archipels et îles), les communautés ont construit des "terroirs maritimes ", conçus, gérés, contrôlés collectivement, en prolongement des terroirs agro-sylvopastoraux, selon les mêmes institutions. Ce sont des espaces circonscrits, bornés (physiquement ou, du moins, socialement), aux droits d'accès et d'usage différenciés. Les terroirs maritimes comprennent des lieux d'exploitation - rizières, pâturage, haut-fond pour la cueillette, pêcherie.., etc. - mais aussi des voies de communication (les chenaux de marée ou bolons), des sites sacrés, des réserves foncières. Ainsi, le terroir maritime, quoique délimité, n'est pas figé mais participe de la dynamique des socio-écosystèmes.

12 Les espaces océaniques, en revanche, se prêtent mal à l'enfermement dans un cadastre géométrique, au découpage en zones homogènes, contiguës et continues, séparées par des limites. Ces espaces ouverts, aux limites perméables et extensives, ne sont néanmoins pas indifférenciés et librement accessibles à tous. Ils font l'objet d'un partage entre les communautés maritimes. Ils sont structurés par des lieux et des itinéraires, constituant autant de territoires en réseaux ou parcours. Le parcours maritime désigne ainsi un espace de migration, fait d'itinéraires et de réseaux sociaux et spatiaux, articulés par des routes autour de nœuds stratégiques (lieux de pêche, débarcadère...) contrôlés par une communauté qui en défend l'accès et les passages. Même si certains espaces halieutiques sont officiellement ouverts et accessibles à tous, les compétences, les moyens techniques et l'appartenance à une communauté sont des facteurs discriminants dans l'accès aux pêcheries. Les pêcheurs dénomment des lieux de pêche et se transmettent leurs savoirs (les informations sur les fonds de pêche, les comportements des poissons, etc.) au sein de leur communauté.

13 Ainsi, à partir d'une vaste revue de la littérature et de recherches empiriques conduites depuis plus de 30 ans en Afrique de l'Ouest, mais aussi à Madagascar et en Asie (Inde, Thaïlande, Vietnam) (Cormier-Salem 1995b, 2000, [sous presse]), nous distinguons schématiquement deux types de territoires de pêche, le terroir des paysans-pêcheurs et le parcours des marins-pêcheurs. Dans le cas du Sénégal, leurs équivalents en wolof sont 
gox pour désigner le terroir maritime, diaar-diaar/yon (ou routes) pour le parcours ; en Basse Casamance, chez les communautés joola, le terme mof ou mofovi est utilisé pour désigner le terroir.

\section{L'institutionnalisation des terroirs maritimes au Sénégal}

14 Quels que soient les termes utilisés pour désigner ces constructions territoriales, soulignons qu'ils correspondent à des institutions fort anciennes, reconnues à l'échelle locale ou régionale, voire globale, et répandues partout dans le monde (Cormier-Salem sous presse), de la Méditerranée avec les prud'homies (Braudel 1949) aux fiefs halieutiques de Calabre en Italie (Collet 1985), des « lobster gang» du Maine aux États-Unis (Acheson 1975) aux tenures maritimes des îles pacifiques (Ruddle \& Akimichi 1984). En Afrique de l'Ouest, cette diversité et cette flexibilité des territoires halieutiques sont bien mises en évidence par divers auteurs, aussi bien en milieu maritime (Pliya 1985, Verdeaux 1992, Chauveau et al. 2000) qu'en milieu fluvial (Fay 1989, Gallais 1967, Croix 2015).

La communauté villageoise, dont l'existence dépend, entre autres, de l'usage des ressources halieutiques et qui en a l'usufruit, assure sa reproductibilité, en ajustant, réaménageant et créant les droits coutumiers en fonction des modifications de l'environnement. Ainsi, plusieurs modèles de gouvernance territoriale ont pu être observés au sein des communautés de pêcheurs artisans au Sénégal. Traditionnellement, les règles d'accès à la ressource étaient généralement différentes d'une localité à une autre. Le milieu marin était divisé en territoires ou aires de pouvoir, dont l'exploitation et la gouvernance incombaient à des lignages ou à des villages. Ainsi, la tutelle exercée par les villages sur les aires de pêche faisait qu'il était strictement interdit à tout étranger de pêcher dans les eaux adjacentes sans autorisation préalable du chef de village. L'ouverture de la pêche était annoncée hors du terroir pour informer tous les ressortissants des villages voisins. Ces pêcheurs devaient alors se présenter aux notables avant de bénéficier du droit de pêche. Ils devaient respecter les règles en vigueur dans le village et offrir une part de leurs captures aux autorités coutumières locales. Ceux qui enfreignaient cette règle encouraient un châtiment sévère (Diouf et al. 1998).

Exceptionnellement, des pêches pouvaient être pratiquées durant la période de fermeture pour des raisons sociales particulières. Ainsi, le chef de village et son Conseil pouvaient autoriser une pêche à une famille ayant un décès, afin de pouvoir utiliser une partie des captures pour les repas funéraires et le revenu de la vente des produits restants pour couvrir les autres frais. Il en était de même pour une famille ayant subi des pertes importantes suite à un incendie ou une autre catastrophe.

Dans certains villages des îles du Saloum, où les pêcheurs étaient aussi agriculteurs, il y avait une répartition saisonnière stricte des activités : la pêche était interdite, sauf dans les bolons (ou chenaux de marée) très en amont, pendant la période d'activité agricole (hivernage ou saison des pluies) et n'était ouverte qu'en fin de saison des pluies en septembre. Cette tradition permettait de protéger le stock d'adultes pendant la période de reproduction.

18 Il en est de même en Casamance, où les communautés de paysans-pêcheurs contrôlent des terroirs aquatiques, structurés par l'exploitation de la mangrove et des bras du fleuve, mais aussi par la riziculture, l'élevage, la cueillette des fruits de la brousse et des 
palmeraies, etc. Nos relevés de terrain, réalisés avec une approche de cartographie participative, ont permis de dresser une carte de ces terroirs à l'échelle des communautés villageoises et de la région de Ziguinchor, révélant l'emprise des communautés sur des espaces divers, du fleuve Casamance et des bolons aux plateaux, ainsi que la complexité des formes de tenure en fonction des systèmes d'exploitation et de l'environnement (Cormier-Salem 1992) (Figure 1). De fait, les règles d'usage et d'accès ne sont pas fixes, mais renégociées entre acteurs et adaptées aux changements des conditions d'exploitation. Ainsi, à l'échelle de la communauté (qui correspond généralement à un clan) chaque quartier (ou lignage) a ses zones de cueillette des huitres, ses débarcadères et ses chantiers pour transformer les huitres. Certaines zones de mangrove sont mises en défens, le temps que les huitres, qui s'accrochent aux racines de palétuviers, se développent à nouveau. En saison des pluies, période de reproduction des huitres, la cueillette est interdite.

Figure 1 : Carte des terroirs et pays de Casamance, Sénégal

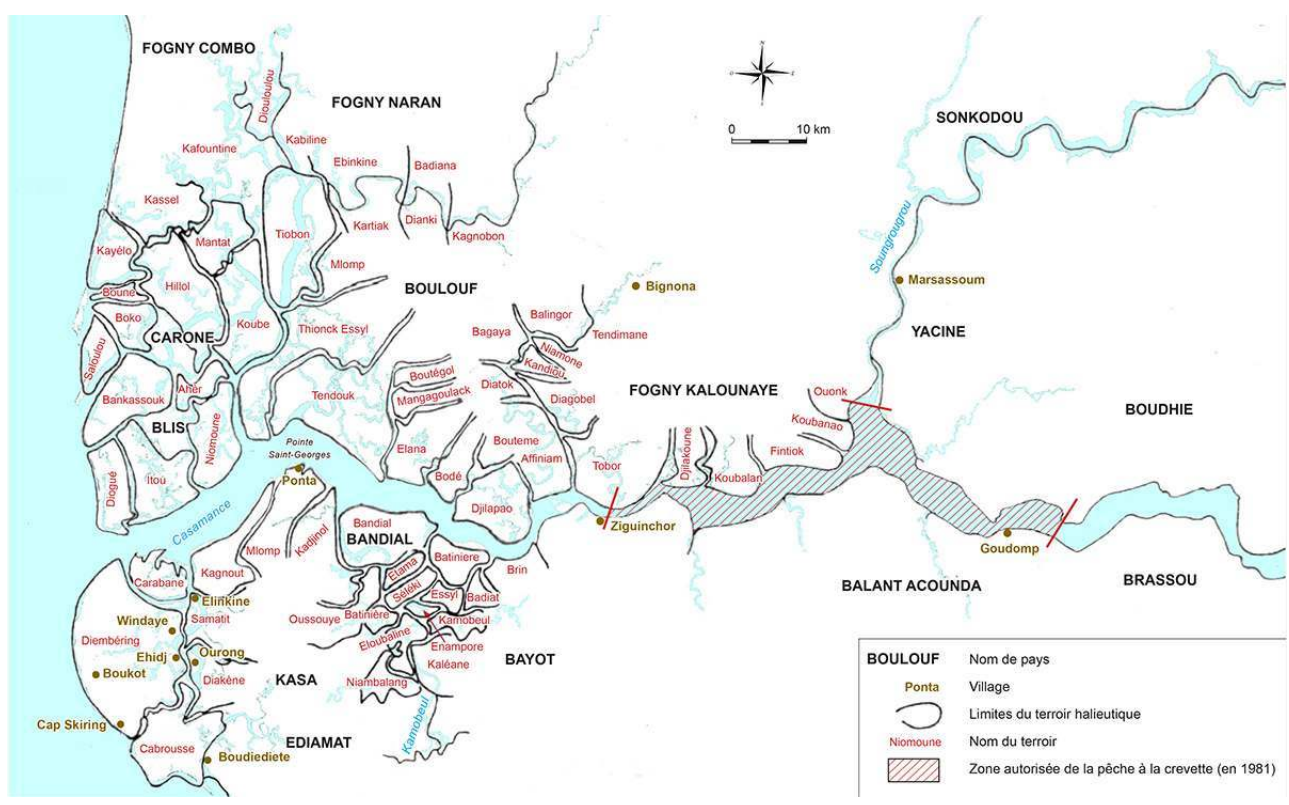

Sources : enquêtes Cormier-Salem, 1984-87 in Cormier-Salem 1992

En outre, les communautés de pêcheurs ont toujours associé une forte charge symbolique à l'espace marin. Le terroir maritime d'un village ainsi que ses habitants sont protégés par des génies, le plus souvent appelés Maam (textuellement « la grand-mère ») auquel est attribué un nom propre. On peut ainsi citer: Maam Ndare résidant à l'île de Yoff; Maam Ndëk Davër ou Ndëk Daour résidant à Daveur ou Veur (îles de la Madeleine); Maam Coumba Castel résidant à Gorée et au plateau de Castel; Maam Coumbame Lambe résidant à Ndépé, à Rufisque, Maam Coumba Bank à Saint-Louis, etc. Dans certains endroits du terroir maritime, les ressources, qu'on trouve, ne peuvent être consommées ou utilisées que sur place. Elles ne doivent en aucun cas sortir de ce terroir. Tout objet, même une pierre, amené sur le continent sera réclamé dans la nuit à celui qui l'a transporté et qui doit le ramener à sa place, faute de quoi la punition du génie peut lui être fatale (Fall 2006). Même dans les terroirs maritimes, où la pêche est autorisée, des contraintes peuvent être imposées. Par exemple, tout poisson pris et dont l'espèce n'est 
pas connue, doit être relâché. Cette pratique traduit, entre autres préoccupations ${ }^{6}$, celle d'assurer la reproduction et la conservation des espèces rares ou en voie de disparition.

Ces règles et croyances se sont construites au fil du temps sur la base de valeurs socioculturelles propres à chaque communauté sur son terroir maritime. Elles sont admises depuis des générations et sont respectées par tous. Ces terroirs maritimes ne font pas l'objet d'un marquage, du moins fixe, ni d'un cadastrage, ou enregistrement écrit. Ils relèvent de la tradition orale, gouvernés par des règles coutumières et des institutions locales, souvent qualifiées d'informelles, mais qui n'en sont pas moins reconnues et respectées par les populations, résidents locaux comme migrants. On pourrait multiplier de tels exemples. Soulignons que cette maitrise coutumière de l'espace maritime a été un des outils les plus efficaces de préservation de la ressource (Berkes et al. 1989, Ruddle et al. 1992). Finalement, on constate que la relation particulière instaurée entre les communautés de pêcheurs artisans sénégalais et l'espace maritime qu'ils exploitent, contrôlent et dans lequel ils s'identifient, considéré donc comme leur terroir, contribue à protéger et pérenniser la ressource halieutique.

\section{Chronique de la fin (officielle mais non réelle) des terroirs maritimes?}

La fin des terroirs est une expression inspirée de l'ouvrage de Weber pour désigner la fin du monde paysan en France au XIX ${ }^{e}$ siècle et, dans notre étude, la crise des systèmes agraires, y compris la crise des systèmes de pêche, et la remise en cause des systèmes anciens sous l'effet combiné de divers facteurs, biophysiques et socio-politiques. À partir des années 1950, l'exode rural, et l'accélération de l'urbanisation et de la littoralisation, la sécheresse, qui sévit en Afrique sub-saharienne des années 1960 aux années 1990, les nouveaux régimes de droits, nationaux et internationaux, modifient profondément les systèmes de pêche (Cormier-Salem 2000). Si de nombreux auteurs en sciences sociales s'accordent à reconnaître la pertinence et l'efficience sur la longue durée des systèmes de tenure traditionnels, ils soulignent également leur fréquente désuétude actuelle et les dysfonctionnements, qui conduisent à des conflits (Ostrom 1990, Péluso 1993).

Avec la colonisation, puis l'Indépendance des États, la mise en place de dispositifs publics centralisés et l'élaboration d'une législation officielle, souvent inspirées par le droit romain, imposent de nouveaux régimes de droits dits positifs. Les nouvelles allocations des ressources et des espaces maritimes sont également inspirées par l'économie néoclassique et le néolibéralisme qui défendent la thèse selon laquelle la propriété commune de la ressource est le facteur explicatif déterminant des problèmes du secteur des pêches. Toujours selon cette conception, qui suppose qu'il n'existe pas de limite d'accès à la ressource, celle-ci serait exploitée par des acteurs (pêcheurs) qui ne se soucient guère des effets mêmes négatifs de leurs actions sur les conditions de pêche d'autres acteurs supposés être leurs concurrents dans l'exploitation de la ressource. Il découle de cette mauvaise allocation de l'effort de pêche, la dissipation de la rente qui aurait pu être générée par le site de pêche. Ainsi, l'idée principale de l'approche des économistes néoclassiques tel Gordon $(1953,1954)$ est basée sur ce constat : la propriété commune de la ressource est la cause de la dissipation de la rente qu'aurait pu générer une pêcherie donnée, et il en découle la pauvreté universelle des pêcheurs. 
23 Pour les théoriciens de l'économie politique, pour générer un rapport de rente économique dans la pêche, il faut contrôler l'accès à la mer, autrement dit aux sites de pêche; Cette vision orientée vers l'attribution de droits de propriété trouve son fondement dans la théorie de la tragédie des communaux de Hardin (1968). La théorie de Hardin, couplée à une vision occidentale sur les sociétés des pays du Tiers Monde, a eu des répercutions notables sur la gestion des ressources.

24 C'est ainsi que face à l'irrationalité supposée des populations des sociétés non occidentales et plus particulièrement des populations rurales, les États indépendants, non encore débarrassés de la vision de l'État colonial, ont voulu s'approprier les ressources naturelles pour en garantir une gestion « rationnelle » (Lavigne Delville $2001: 5$ ).

Ainsi, sur la conception que le contrôle de l'État constitue la seule façon de prévenir la dégradation des ressources, le Sénégal a institué par la loi du 17 juin 1964, le Domaine National (Bonnin et al. 2016), De ce fait dans ,le premier code de la pêche élaboré dans les années 60 dont les grandes lignes ont été reproduites dans la loi No $98-32$ du 14 avril 1998 définissant le nouveau code de la pêche révisé, il a été institué que :

« les ressources halieutiques sous juridiction sénégalaise constituent un patrimoine national. Le droit de pêche dans les eaux maritimes sous juridiction sénégalaise appartient à l'État qui seul peut en autoriser l'exercice à des personnes physiques ou morales de nationalité sénégalaise ou étrangère. La gestion des ressources halieutiques est une prérogative de l'État ».

26 La promulgation de cette loi instituant une gestion étatique centralisée a mis à mal les pratiques traditionnelles de tenures maritimes en dépossédant les communautés de pêcheurs des droits coutumiers qu'elles exerçaient sur leurs terroirs et parcours.

Les mesures prônées par les agents de l'État dans les politiques de gestion relèvent d'une vision techniciste de gestion, peu soucieuse des considérations socio-anthropologiques des connaissances des communautés de pêcheurs. Les règles étatiques de gestion ainsi instituées, n'ayant pas pris en compte les savoirs et savoir-faire des communautés, sont entrées en contradiction avec les représentations que celles-ci se font des espaces halieutiques et des ressources. La conséquence de cette vision se répercute sur une législation étatique jugée inadaptée et des mesures réglementaires inappliquées soit par manque de moyens de contrôle, soit par ignorance de ces mesures par les pêcheurs. À titre d'exemples, certaines techniques de pêche, interdites par le code (cf. interdiction fixée par l'article 30 du décret d'application du code), sont toujours utilisées, tels les filets maillants fabriqués à partir d'éléments monofilaments et multimonofilaments ou encore les matières explosives et substances ou appâts toxiques. La taille réelle des mailles de filet, notamment de grande prise, est souvent inférieure à la taille légale définie par le code et les critères de mesure de l'écart des mailles entre elles ne sont pas respectés. Les limites légales de la zonation des espaces de pêche réservés au secteur artisanal sont souvent méconnues par les pêcheurs artisanaux. Les zones maritimes interdites de pêche et de mouillage ne sont pas assurées. Les repos biologiques sur certaines espèces ne sont pas respectés.

\section{Le renouveau des terroirs maritimes}

En contre-point au néocolonialisme et à l'impérialisme vert, au globalisme et au néolibéralisme, depuis les années 1990, on assiste à un retour en force du local. Ce retour 
s'exprime notamment par la réhabilitation des communautés locales comme garantes de la préservation du jardin planétaire et le renouveau des terroirs maritimes.

Plusieurs motivations, politiques, éthiques, instrumentales, heuristiques (Demeritt 2015), justifient le changement de paradigme en termes de gouvernance des mers. Le regain d'intérêt pour les savoirs locaux et la gestion communautaire de la part des organismes internationaux sont explicites à partir des années 1990, suite au rapport Brundtland, popularisant la notion de développement durable. La convention de Rio sur la biodiversité (1992), dans l'article 8 alinéa J, tout en reconnaissant la souveraineté des États sur leur territoire, rend nécessaire la prise en compte des «savoirs, traditions et innovations des populations indigènes et peuples autochtones" (Cormier-Salem \& Roussel 2002). Cet article ouvre la voie à une série de mesures et règlements qui débouchent notamment sur le protocole de Nagoya (2005). L'échec des instruments coercitifs et la montée du participatif ont ainsi des justifications éthiques, bien exprimées à travers le succès des notions de partage des bénéfices, solidarité écologique et sociale, justice environnementale, mais aussi opérationnelles, ces mesures étant considérées comme plus efficaces (Borrini-Feyerabend et al. 2009).

Ainsi au Sénégal, l'échec des politiques publiques et l'incapacité de l'État à faire respecter la réglementation officielle, la remise en cause par les communautés locales des normes et mesures de gestion de l'État sénégalais (illégitime, inefficace, injuste, impopulaire) et, enfin, le doute sur les expertises scientifiques, à la base de ces politiques, justifient le renouveau des terroirs maritimes.

31 Parallèlement au développement de théories mettant à nue la conception néoclassique de la gestion des ressources (MacCay et al. 1987, Berkes et al.1989, Ostrom 1990, Ostrom et al. 2002). Selon Ruddle \& Akimichi (1984), la gestion étatique ou gestion «moderne«s'est très vite révélée inadaptée (Compagnon \& Constantin 2000, Ribot et al. 2006, CormierSalem sous presse). Ainsi, au Sénégal, les politiques publiques, en matière de réglementation du secteur de la pêche artisanale, ont suscité de forts mécontentements de la part des communautés de pêcheurs, soit parce que certaines mesures à l'élaboration desquelles ils n'avaient pas été associés, heurtaient leurs convictions socioculturelles, soit parce que l'administration n'assurait pas sur le terrain la mise en application de décisions pourtant jugées pertinentes par les pêcheurs eux-mêmes. Cette situation a poussé certains villages de pêcheurs à s'organiser au niveau de leur localité (village ou village) au début des années 1990 pour imposer des mesures de gestion dans leur terroir maritime.

Ainsi, à Cayar sous l'autorité du chef de village, les populations ont mis en place une instance dénommée Comité de pêche de Cayar (CPC) en 1994, À travers cette instance ce sont les pêcheurs eux-mêmes qui détruisent les filets dormants en monofilament, interdits par la réglementation et tolérées par les représentants de l'Administration sur le terrain. Les pêcheurs ont obtenu la délimitation de la zone de pêche réservée aux filets dormants, l'interdiction de l'utilisation des palangres sur les rochers, l'instauration d'une seule sortie par jour pour les sennes tournantes, l'instauration d'un quota de trois caisses par pirogue et par jour pour les captures de certaines espèces (dorade, pageot, pagre), l'interdiction de l'utilisation des filets à monofilaments, l'instauration d'une taxe au débarquement sur les pirogues.

33 À Yoff, il a été noté que c'est dans ce centre que les premières mesures concernant la réglementation des techniques de pêche auraient été prises dans les années 1990 sur l'initiative de l'instance traditionnelle appelée ferey. Le ferey est une intance lebu en 
charge de toutes les affaires du village, à l'instar du conseil municipal et constituée par des notables représentatifs des sept penc ou quartier du village. Ces mesures ont commencé par la limitation du nombre de caisses débarquées pour certaines espèces pêchées à la ligne. D'autres mesures ont été ensuite mises en place pour essayer de réglementer toutes les activités de pêche dans le centre. Elles concernent principalement, la prohibition de l'utilisation des filets dormants, l'interdiction pour les sennes tournantes de pêcher à une certaine distance de la plage afin de permettre aux sennes de plage de se déployer librement, surtout durant les périodes d'affluence de pirogues entre février et mai, la rotation des sorties pour les sennes de plage par manque d'espace, l'interdiction de l'utilisation des palangres dans certaines zones entre février et mai, l'interdiction de débarquement de poisson venant d'autres centres de pêche dans le but d'éviter une surproduction qui pourrait entraîner la baisse de prix, l'instauration d'une taxe de 500 FCFA aux mareyeurs pour chaque chargement effectué à partir de la plage de Yoff.

À Mbour, les pêcheurs ont mis en place en 1992 une instance dénommée Baatu Tefes (textuellement : la voix ou la parole de la plage) qui a élaboré un code de conduite des activités de pêche dans le centre. Cette institution qui a obtenu sa reconnaissance auprès des autorités municipales et administratives notamment la Préfecture et le Service des pêches a pris un certain nombre de mesures dont l'application a connu plus ou moins un succès. Il s'agit, entre autres mesures, de l'interdiction de l'utilisation de la palangre, l'interdiction des sorties nocturnes pour les sennes tournantes et les filets dormants pendant certaines périodes de l'année, l'interdiction de la pêche au fusil, l'instauration d'une taxe au débarquement sur les pirogues, l'interdiction de la pêche des juvéniles.

Dans le Saloum, il a été noté qu'à Bétenty, sous l'autorité du chef de village, les populations ont convenu que la pêche est fermée pour tous les pêcheurs dans la zone dénommée «Passe de Bétenti » qui comprend la zone côtière de la façade maritime mais aussi les principaux chenaux environnants. Les activités de pêche ne sont permises que dans les bolons situés plus à l'intérieur où l'utilisation des sennes de plage n'est pas autorisée. La pêche n'est réouverte qu'en saison des pluies vers le début du mois de septembre. il a été aussi institué une interdiction de la pêche à la crevette durant les période de mortes eaux, appelée Niokok en langue niominka et dont l'initiative porte d'ailleurs le nom.

À Soucouta et les villages environnants, les populations locales ont entrepris des actions allant dans la sens d'une meilleure exploitation des ressources dans la zone. C'est ainsi qu'il a été mis en place un comité inter-villageois réunissant les sept villages qui ont pris comme mesures, d'interdire la pêche dans le bolon de Bamboung pendant une certaine période de l'année, d'interdire définitivement l'utilisation de la palangre, des filets dormants, des sennes de plage et la pratique des marées, d'instaurer une taxe sur les pirogues opérant dans la zone.

Au début des années 2000, les autorités publiques (service des pêches, des eaux et forêts, des parcs nationaux) ont commencé à accompagner ces initiatives en formalisant, par arrêté de l'autorité administrative locale, certaines mesures, même lorsqu'elles étaient seulement basées sur les connaissances empiriques des professionnels.

Cette attitude des autorités montre que les institutions étatiques peuvent aussi accompagner les pratiques locales (Mbaye 2013); elle relativise le clivage droit officielpratiques locales, malgré tout dominant. Une nouvelle démarche dans la gestion des ressources, prenant en compte les connaissances des professionnels, est ainsi entreprise 
depuis quelques années. Cette démarche est basée sur une approche volontariste, centrée sur un territoire restreint et qui conçoit la gestion comme partant du bas et en privilégiant les ressources endogènes. Cette démarche fait appel aux traditions et savoirs locaux et insiste particulièrement sur la prise en compte des valeurs culturelles et sur le recours à des modalités de réglementations émanant de la population locale.

Évidemment, aucune de ces formes de gestion n'avait été prise en compte dans le système national de législation des pêches au moment de l'élaboration des différents textes réglementaires. Actuellement, en revanche, les pêcheurs proposent une réglementation sur la base de leurs propres savoirs, qui enrichissent ainsi le droit positif. Les communautés locales avancent ainsi de solides arguments sur la biologie des espèces, les conditions hydrologiques, le comportement des engins vis-à-vis de la ressource et des habitats, obligeant l'administration et les ONG à solliciter l'arbitrage des scientifiques.

Dans certains villages, tels Bétenty, Cayar, Foundiougne, Nianing, etc., la communauté locale a mis en place ce qu'elle appelle une commission scientifique chargée de recueillir et de vulgariser les connaissances locales qui sous-tendent leurs initiatives. Elle positionne cette instance comme l'interlocutrice des scientifiques et interpelle ces derniers pour des recherches participatives de validation de leurs savoirs. Les communautés ont aussi mis en place des comités de surveillance et veulent assurer le contrôle et l'application de la réglementation. Elles sollicitent simplement un appui logistique (pirogue motorisée et carburant notamment) auprès de l'administration et des autres partenaires (ONG, programmes, projets). L'administration s'est donc vue allégée de certaines charges, qui lui incombaient auparavant.

Dans certains cas, comme à Bétenty et à Cayar, avant même l'intervention des scientifiques, l'administration semble reconnaître la pertinence des dispositifs locaux et se dit disposée à officialiser le cadre réglementaire local. Cela a suscité un cadre de concertation entre pêcheurs, administration, recherche et ONG dans la gestion des ressources. Ainsi, la cogestion des ressources, définie comme le processus de la prise de décision concertée et de responsabilités partagées entre les représentants des groupes d'utilisateurs, les organismes gouvernementaux, les institutions de recherche et autres partenaires, suscite un nouvel espoir. Dans ce contexte de la participation, les communautés locales ont été encouragées par les partenaires extérieurs à travers des programmes et projets qui travaillent directement avec elles ou au travers de nouvelles instances locales qu'ils ont mises en place.

Ainsi, l'UICN a mis en place des Comités de plage au Saloum en 2000 ; la JICA, coopération japonaise, a travaillé avec des comités villageois sur la Petite Côte à partir de 2003 ; le programme de Gestion Intégrée des Ressources Marines et Côtières (GIRMAC), lancé en 2003, travaille avec les comités locaux de pêche (CLP); l'USAID/COMFISH a tenté de mettre en place ce qu'elle appelle, des Unités de Gestion durable (UGD). Le succès de tous ces projets est conditionné par l'implication des locaux dès leur conception, puis tout au long du processus de mise en œuvre des actions collectives. C'est ce qui conduit à la mise en place de ces instances locales et de leur reconnaissance officielle à travers des conventions locales.

Tous ces programmes et projets ont mené des initiatives de gestion à l'échelle des terroirs anciens. C'est ainsi que des Aires Protégées ont été initiées avec l'UICN au Saloum dès les années 2000 ; le GIRMAC a initié la mise en place de Zone de Pêche Protégées (ZPP) sur la Petite Côte à partir de 2003 ; l'ONG WWF a initié l'officialisation des premières AMP par l'État en 2005 ; l'ONG Océanium, soutenu par le FEM, a suscité et encouragé la mise en 
place en Casamance ce qu'elle appelle des Aires du Patrimoine Autonome Communauté (APAC) (Cormier-Salem 2014). La JICA, à travers le label OVOP « One Product, One Village », soutient les démarches de qualification des produits de la mer et de la mangrove au Sénégal à partir de 2011, reconnaissant l'excellence des produits de meroir, mais plus encore la solidité des groupements de productrices (Cormier-Salem sous presse b).

Dans ce contexte, l'État sénégalais a institutionnalisé la gestion à l'échelle des terroirs par la réactivation du Conseil Local des Pêches maritimes et la création de la Direction des Aires Communautaire (DAC) au sein du Ministère de l'Économie Maritime.

\section{Les zones de pêche protégées de la Petite Côte au Sénégal}

Les ZPP de la Petite Côte (Figure 2) nous servent d'illustrations de ces processus de retour vers les terroirs maritimes. Les villages de Mbaling, Nianing, Pointe Sarène et Mbodiène, situés sur la Petite Côte, ont envisagé d'avoir chacun une Zone de Pêche Protégée (ZPP) sur leur terroir. Une ZPP est une zone interdite de pêche ou à accès limité ou réglementé, adoptée par les communautés locales dans le cadre d'un système de cogestion faisant l'objet d'accord avec les services compétents de l'État.

Figure 2 : Carte de la Petite Côte, Sénégal

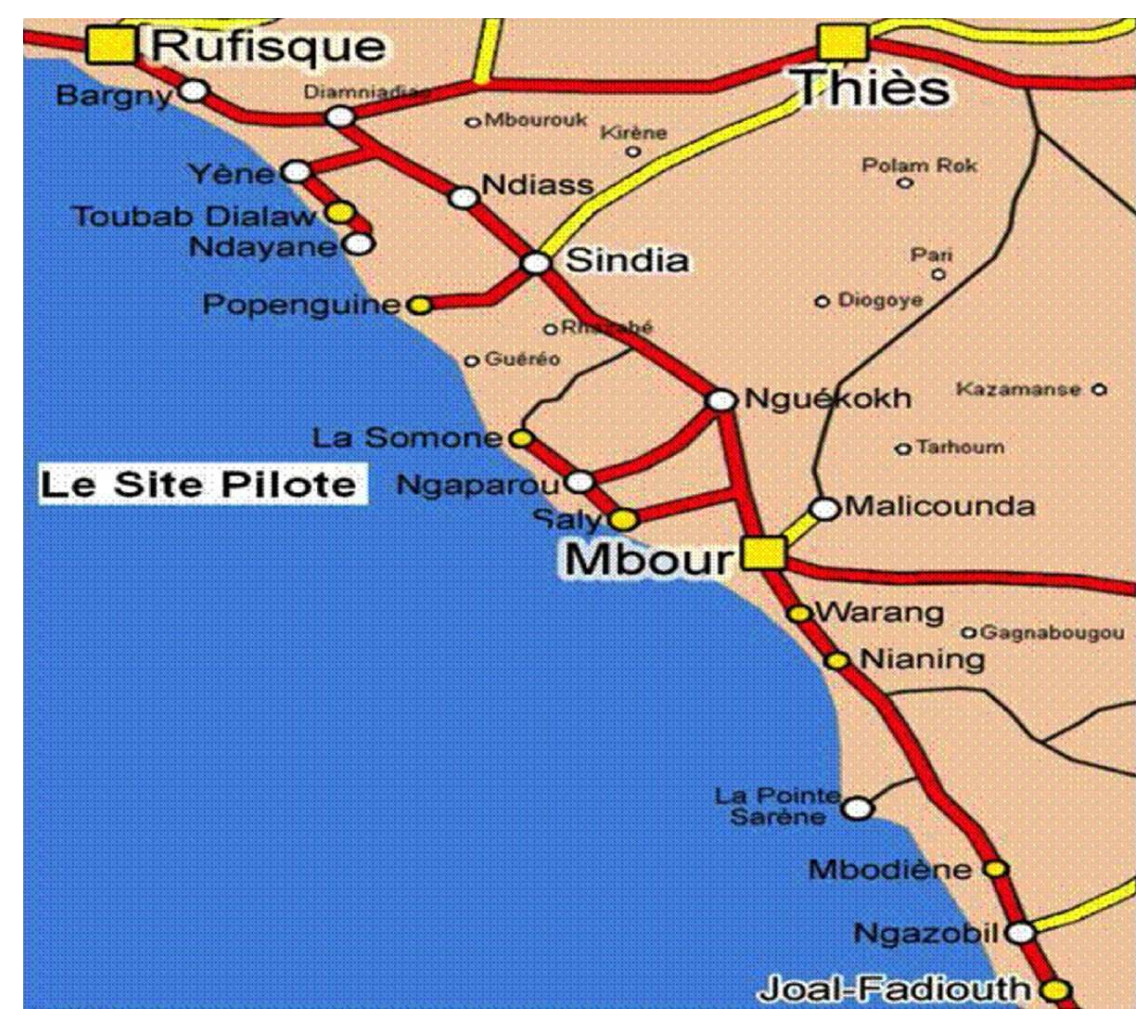

Les pêcheurs définissent les ZPP sur la base des savoirs empiriques qu'ils ont de la biologie des espèces, mais aussi et surtout des moyens dont ils disposent pour assurer une application des règles de gestion sur la zone. Les superficies couvertes vers le large ne dépassent pas ainsi deux kilomètres. Les populations ont fixé la largeur des ZPP à deux kilomètres pour des raisons de capacités de surveillance. Quant à la longueur de chaque 
ZPP, elle a été fixée aux limites du terroir de chaque village par rapport aux autres villages. Chaque village circonscrit les limites de son terroir maritime aux limites de son terroir terrestre. Ces limites ne sont pas matérialisées par des bornes, mais néanmoins reconnues par tous et transmises dans la mémoire collective. Cet espace délimité est appelé « aire de cogestion » qui matérialise l'espace que la population est censée gérée en partenariat avec l'administration des pêches. Les recherches d'accompagnement montrent que l'étroitesse des zones couvertes au large pose le problème de la pertinence des ZPP quant à leur impact réel sur la gestion des espèces démersales ciblées. Sur la Petite Côte, les zones rocheuses de deux à trois mètres de profondeur se trouvent à trois voire quatre kilomètres au large. Ainsi, il est recommandé aux pêcheurs d'avoir une ZPP commune allant jusqu'à trois kilomètres au moins pour couvrir la zone de frayère et de nurserie des principales espèces ciblées. Toutefois, l'argument scientifique mettant en avant le territoire du poisson se heurte à l'identification des communautés à leur terroir. Se pose ainsi la question de l'adéquation entre gouvernance locale et ingénierie écologique.

Le schéma d'aménagement découpe les zones de pêche protégées en zones à exploitation réglementée (ZER) et en zones interdites de pêche (ZIP). Dans certains cas, ce sont deux ZER qui sont aménagées avec une réglementation alternée entre les deux selon le nombre des pêcheurs ou les saisons de pêche. Dans ces conditions, l'une des deux ZER peut être érigée en une ZIP pour permettre à la ressource de se reproduire et/ou de grossir en toute quiétude (Tableau 1 et Figures 3 à 7 )

Tableau 1: Étendue et schéma d'aménagement des aires de cogestion sur les sites

\begin{tabular}{|c|c|c|c|}
\hline Sites & Etendue des ZPP (aire de cogestion) & \multicolumn{2}{|c|}{ Schéma d'aménagement des ZPP (aire de cogestion) } \\
\hline Mbaling & $6 \mathrm{~km}^{2}$ (longueur $3 \mathrm{~km}$ et largeur $2 \mathrm{~km}$ ) & Une ZER de $2 \mathrm{~km}$ de long et $1,16 \mathrm{~km}$ de large & Une ZIP de $1 \mathrm{~km}$ de large \\
\hline Nianing & $15 \mathrm{~km}^{2}$ (longueur $7,5 \mathrm{~km}$ et largeur $2 \mathrm{~km}$ ) & Une ZER de $3.35 \mathrm{~km}$ de large & Une ZIP de $3.32 \mathrm{~km}$ de large \\
\hline Pointe Sarène & $15 \mathrm{~km}^{2}$ (longueur $7,5 \mathrm{~km}$ et largeur $2 \mathrm{~km}$ ) & 2 ZER aux extrémités de la ZPP ( $1,90 \mathrm{~km}$ et $1.41 \mathrm{~km}$ du nord au sud) & Une ZIP de 1.71 de largeur $\mathrm{km}$ \\
\hline Mbodiène & $10 \mathrm{~km}^{2}$ (longueur $5 \mathrm{~km}$ et largeur $2 \mathrm{~km}$ ) & Une ZER de $2.10 \mathrm{~km}$ de large & Deux ZIP aux extrémités et une ZER ( 1.87 et $1.05 \mathrm{~km}$ ) \\
\hline
\end{tabular}

Figure 3 : Délimitation des ZPP, Petite Côte, Sénégal

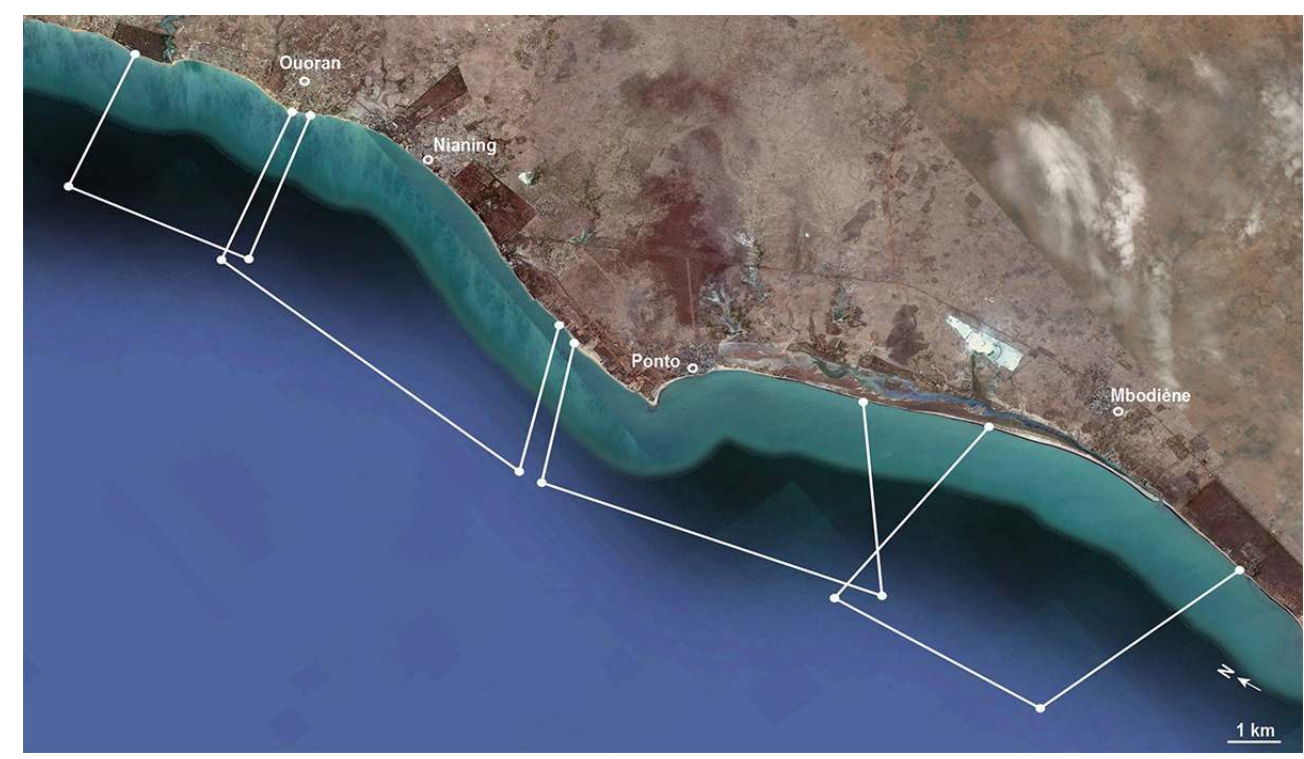


Figure 4 : Schémas d'aménagement de la ZPP de Mbaling

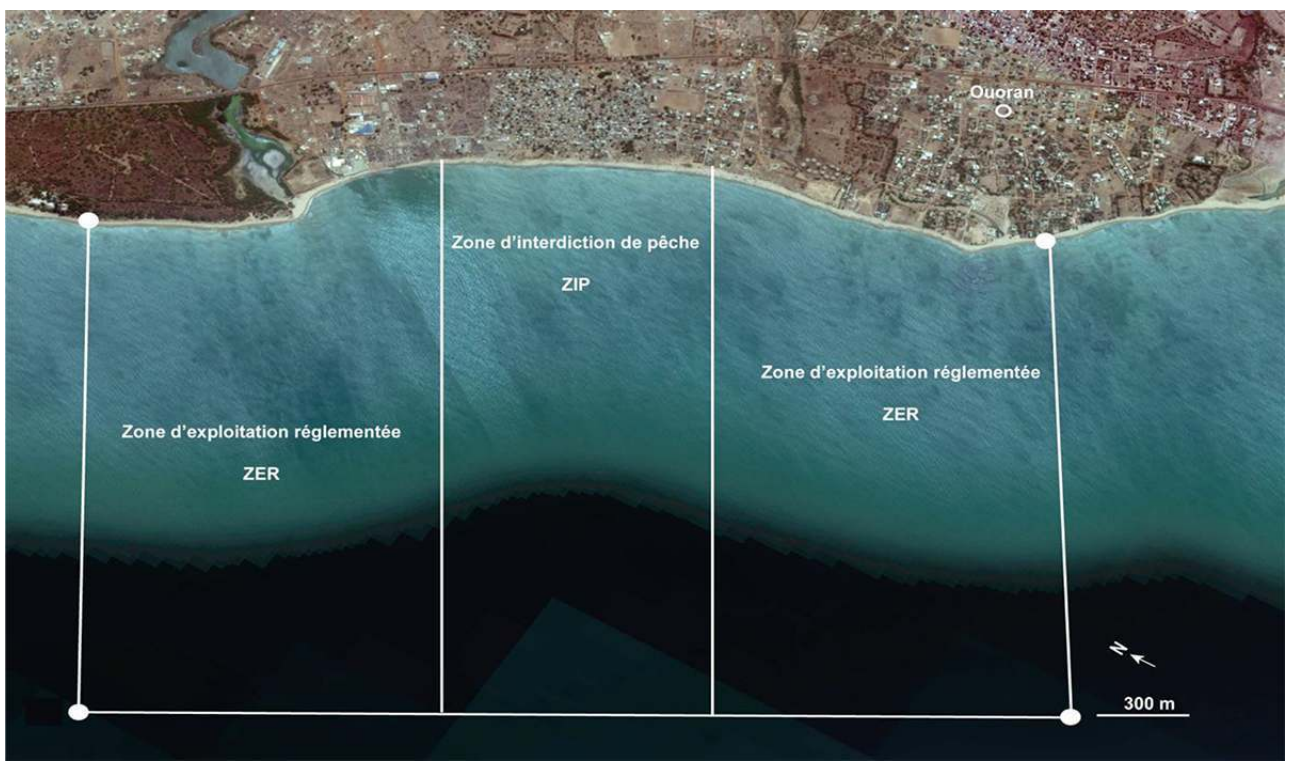

Figure 5 : Schémas d'aménagement de la ZPP de Nianing

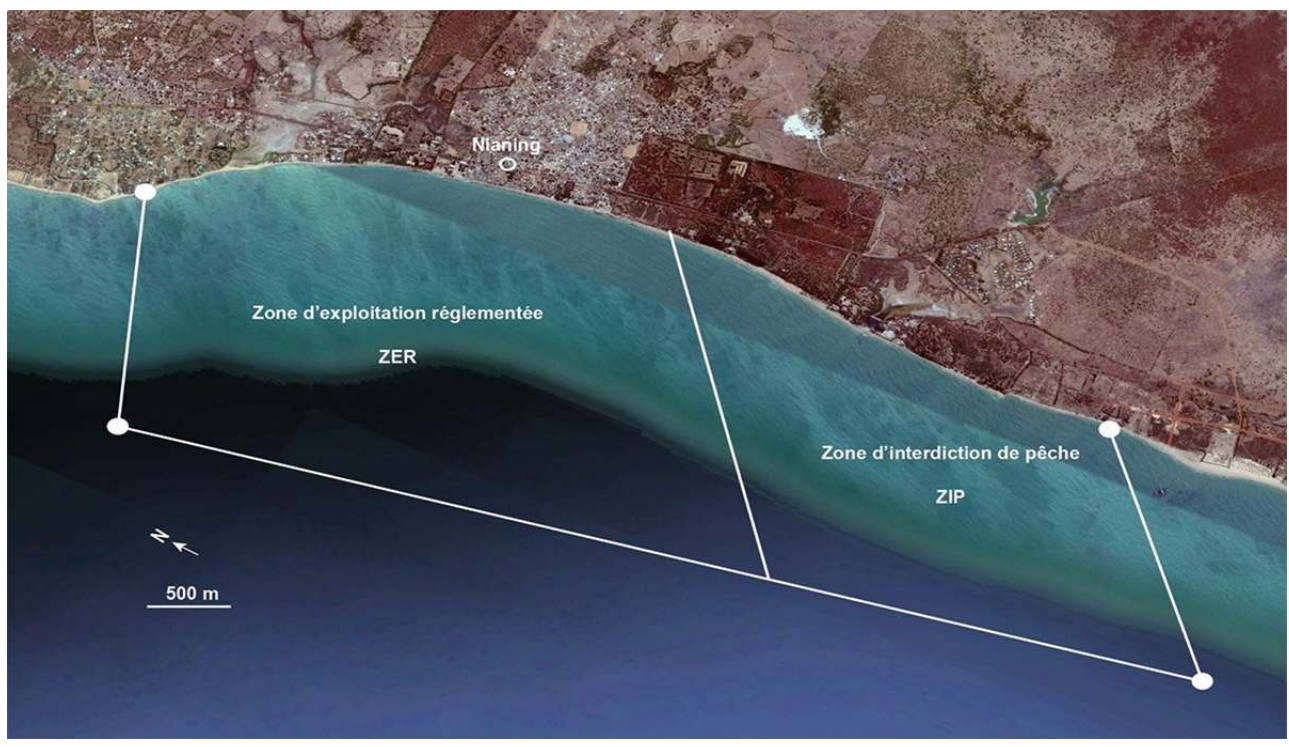


Figure 6 : Schémas d'aménagement de la ZPP de Pointe Sarène

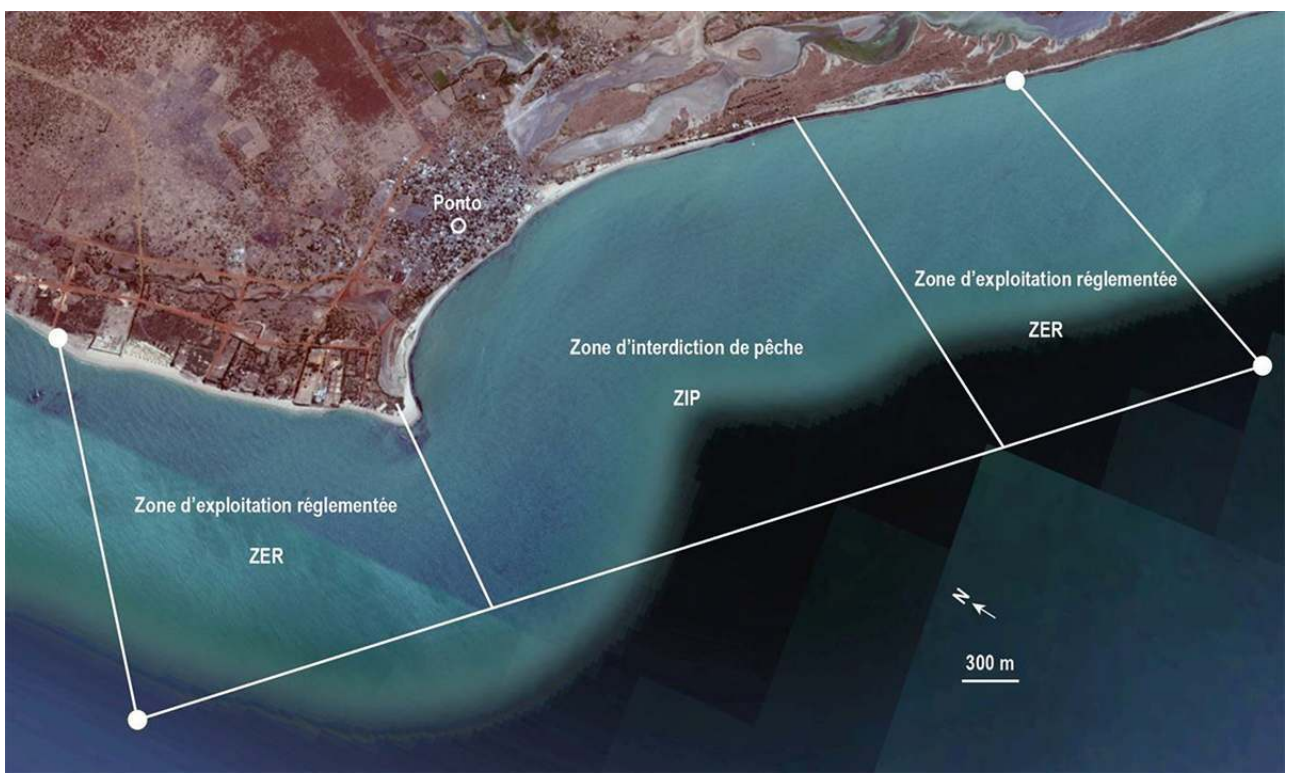

Figure 7 : Schémas d'aménagement de la ZPP de Mbodiène

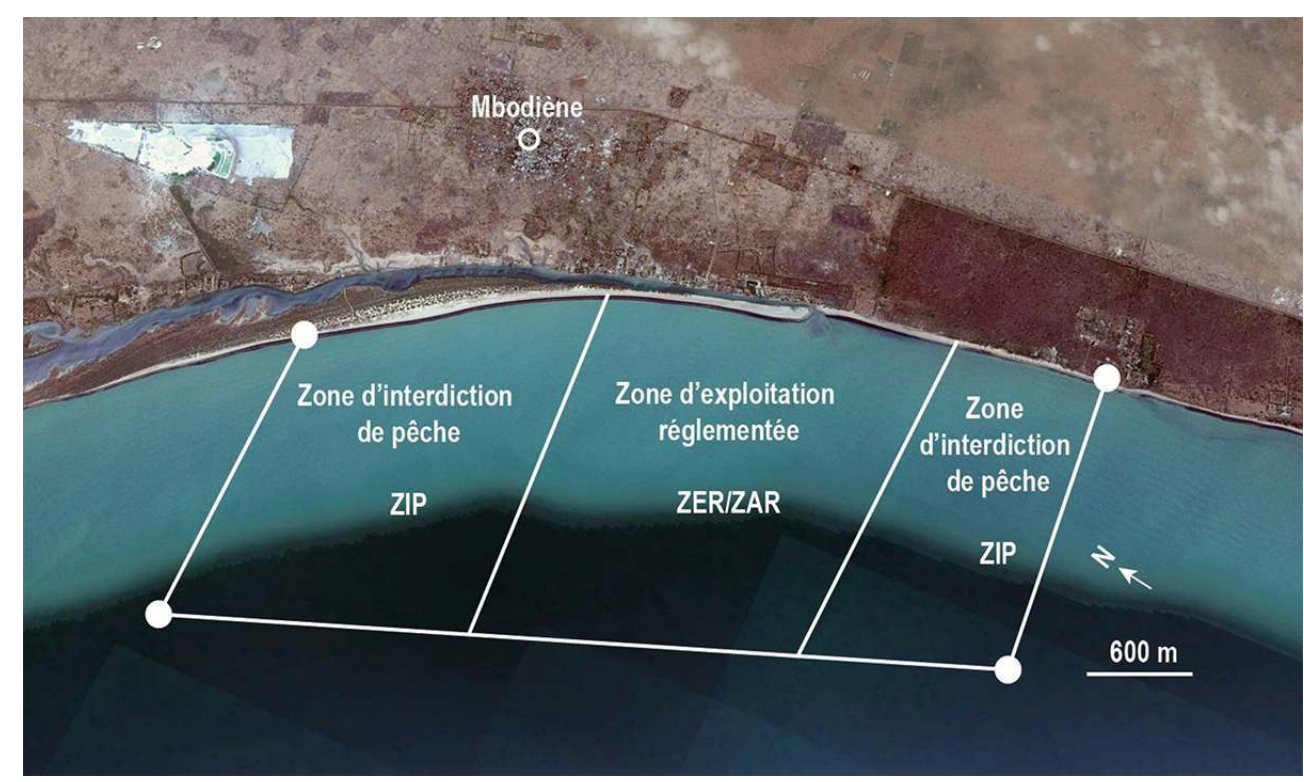

\section{Discussion}

Au Sénégal, nonobstant la diversité des procédures de territorialisation de la mer, il faut souligner les opportunités que ces nouveaux dispositifs (ZPP, APAC, etc.) offrent aux communautés locales : les terroirs, nouveaux ou renouvelés, quelques soient les termes utilisés pour les désigner, sont issus d'un processus de concertation, où les professionnels de la mer sont considérés comme les acteurs de base. Ce sont des arènes de négociation, comprenant une grande diversité d'acteurs et où sont promus les savoirs locaux. Néanmoins, ces processus comportent des limites: la pêche n'est pas encore une compétence transférée. La création de la Direction des Aires Marines Protégées 
Communautaires (DAMPC) au sein du Ministère de l'Environnement, et non au sein du Ministère de l'Économie Maritime pose le problème de la cohésion des politiques publiques (Mbaye et al. 2013). Enfin la communalisation des territoires entraîne le plus souvent la perte de pouvoir du chef du village, autour de qui s'élaborait la gouvernance traditionnelle. Les chefs des lignages et clans sont désormais remplacés par les délégués de quartier cooptés par le Maire, lui-même élu; les conseils des sages ou des anciens remplacés par des Comités de plage, dont les membres sont désignés par les pêcheurs.

L'histoire de la fin ou du renouveau des terroirs maritimes au Sénégal renvoie au débat plus global de la coexistence entre droit positif moderne et règles et pratiques traditionnelles. Avant la période coloniale, la gestion des ressources naturelles et des espaces, tout comme l'organisation politique et économique des communautés rurales, font état de modes de régulation plus ou moins élaborés et discrets, mais néanmoins tacites et respectés par tous, traduisant un sentiment incontestable d'appartenance sociale et culturelle. Ces terroirs maritimes comptent parmi les principaux héritages transmis par les Anciens; ils sont gérés par les chefs de clan et de lignage, afin d'en assurer la préservation et la transmission pour les générations futures. Ce sont des patrimoines, objets d'une forte conscience identitaire et revendiqués comme tels par les groupes locaux à l'encontre des autres acteurs.

Dès l'époque coloniale puis avec les nouveaux États indépendants, les régimes juridiques, basés sur le droit positif, conduisent à ignorer, sinon détruire les systèmes anciens de valeurs, règles et pratiques. Les espaces maritimes, non aménagés de façon permanente, mais collectivement gérés et maîtrisés par les communautés locales, font ainsi l'objet d'accaparements publics et privés (Cormier-Salem 2014).

51 Après des décennies d'impérialisme colonial, de centralisation étatique et de globalisation, on assiste à une remise en cause des systèmes de gouvernance centralisés et descendants. La fragilité de ces systèmes dits modernes ainsi que la faiblesse des institutions publiques sont patentes dans les pays du Sud, conduisant à un redimensionnement des missions de l'État et à la prise en compte des revendications locales. À cet égard, au Sénégal, l'adoption du nouveau Code des collectivités locales, dans lequel le législateur reconnaît les droits et pratiques coutumières et recherche la compatibilité entre us et coutumes et droit positif, est un tournant décisif. La décentralisation, en tant que réforme transversale, consacre le principe de la prise en charge du développement par les populations elles-mêmes (organes de gestion élus, transfert de compétences, etc.) ; les différentes politiques sectorielles se focalisent sur la démocratisation et la responsabilisation dans la gestion des intérêts collectifs. La politique en matière de gestion des ressources naturelles s'est inscrite dans ce cadre, à travers la responsabilisation des collectivités territoriales et des populations sur leurs terroirs. L'appui au développement des partenaires, telles les agences de développement (JICA, USAID, GTZ, AFD) ainsi que la Banque Mondiale, ont initié des projets visant à renforcer la participation des communautés locales à la gestion de leurs espaces et de leurs ressources. Cette option a été affirmée par l'inscription de la cogestion des ressources halieutiques dans le nouveau code de la pêche, basée sur la reconnaissance juridique des organisations communautaires et leur responsabilisation dans la gestion durable des ressources halieutiques. Cette nouvelle démarche de l'administration consiste à inciter les populations locales à proposer des mesures de gestion sur la base de leurs connaissances du milieu et de la ressource. Ces « initiatives locales de cogestion ", selon l'expression consacrée, sont basées sur une approche volontariste, délimitées à un 
territoire restreint et au moyen de démarches ascendantes, faisant appel aux traditions et savoirs locaux, et basées sur des instances anciennes (tels le Conseil des anciens) ou renouvelées (comme les CLPA et les CLP). La prise en compte des valeurs culturelles et le recours à des modalités de réglementations émanant de la population locale constituent le cœur de ces démarches (Mbaye et al. 2016). Afin d'affirmer sa volonté de prendre en compte le local, l'État sénégalais a mis en place une Direction des Aires Communautaires au sein du Ministère en charge de la pêche, devenu actuellement la Direction des Aires marines protégées communautaires.

La pêche étant toujours considérée comme une compétence non transférée, la gestion des ressources halieutiques au niveau local se fait à travers des conventions locales. Celles-ci sont novatrices en ce qu'elles permettent aux communautés locales de négocier les règles à instaurer dans leurs terroirs maritimes avec les administrations (Granier 2006). Toutefois, dans le cas du Sénégal, elles sont perçues aussi comme des outils de gestion des rapports sociaux. Les actions entreprises par les communautés locales reflètent une attitude d'ostracisme à l'égard de certaines catégories d'acteurs perçus comme des concurrents et un désir de les exclure de l'accès à leurs espaces et ressources. C'est ainsi que le renouveau des terroirs n'est pas dénué de risques d'injustice à l'égard des minorités (cadets, femmes, allochtones..). Il peut être porteur de repli identitaire, de fermeture vers l'extérieur et de rejet d'autres groupes sociaux (Dahou \& Abdel Weddoud 2007, Cormier-Salem 2017).

53 Ainsi, bien qu'en capitalisant sur ces succès indéniables en termes de revalorisation des savoirs traditionnels, d'appropriation de la gestion des ressources naturelles par les populations et d'intermédiation à l'échelle des terroirs, les nouveaux champs de recherche en sciences sociales, de l'anthropologie maritime à l'écologie politique, devraient s'orienter vers le développement d'approches méthodologiques et d'outils de travail permettant d'aborder la dimension intercommunautaire des terroir. La problématique du retour au local ou le renouveau des terroirs dans la gestion décentralisée des ressources naturelles comme axe stratégique d'intervention, est le défi lancé aujourd'hui aux différents dispositifs d'intervention, tant du côté de l'État que du côté des partenaires au développement. L'anthropologie maritime a toujours été au cœur des modes d'organisation et de régulation sociale des populations et se doit d'éclairer davantage les institutions stratifiées et complexes existants (chefs de village, responsables de culte religieux, chefs coutumiers, groupes d'âge chargés des missions de surveillance...), de même qu'un ensemble de normes et valeurs qui régissent l'échelle des terroirs et leur inter-connexion, autrement dit les solidarités territoriales, à la fois écologiques et sociales, principes de gouvernance de plus en plus mises en avant à l'échelle nationale et globale (Mathevet et al. 2010, Cormier-Salem sous presse).

\section{Conclusion : terroirs revisités ou mer globale?}

Le succès de la notion de terroir tient à sa richesse sémantique, qui en fait aussi son ambiguïté, et à son fort pouvoir d'évocation, du lien aux lieux (ou places en anglais) au goût du terroir ou meroir dans le cas des produits issus de la mer. La réinvention (ou le renouveau) des terroirs maritimes, longtemps méconnus par les institutions dites formelles, s'ancre dans le passé, les traditions, les systèmes anciens de gouvernance des espaces, mais représente aussi une trajectoire innovante, expression d'un contre-pouvoir à la globalisation de la mer et à la normalisation des règles internationales. Ces fortes 
dynamiques actuelles témoignent de la capacité des communautés locales à se saisir d'opportunités et à élaborer de nouvelles formes de gouvernance. La légitimation par l'État de mesures de régulation locales, à travers l'adoption de conventions locales, ou encore la qualification des produits locaux, témoignent de la reconnaissance des revendications locales, qui sont tout à la fois territoriales, patrimoniales et identitaires, Néanmoins, ces dynamiques ne sont pas sans risques d'essentialisme et de communautarisme, et par conséquent d'injustice à l'égard des " allochtones ", migrants et minorités. Les communautés locales elles-mêmes, ne sont pas homogènes et ne cessent de se recomposer. Certaines actions collectives, initiées par les "locaux", sont issues d'alliances ambigües, ou du moins contractées sur de nouvelles bases, entre élus locaux, ONG et instances de l'État. Ces jeux politiques se font souvent au détriment du pouvoir des Anciens et, comme dans le cas du Sénégal, conduisent à la fragmentation des conseils locaux et des instances traditionnelles.

Ces dynamiques interrogent, enfin, sur les régimes d'autorité, le rôle de l'État ainsi que les poids des ONG et des bailleurs, comme la Banque Mondiale et le FMI, qui imposent leurs valeurs, normes et mécanismes. Comme dans le cas des political forest analysé par Péluso (1993) dans le contexte d'États coercitifs, la mer est devenue un enjeu politique, $a$ political sea, à diverses échelles, des terroirs locaux à la mer globale. Sous couvert de participation et de transfert de gestion aux communautés locales, les politiques publiques, qu'elles soient coercitives ou incitatives, permettent aux États de garder le contrôle sur les hommes et les territoires. La communalisation conduit à la territorialisation étatique. Les terroirs maritimes sont ainsi imbriqués dans un jeu complexe d'acteurs, institutions et normes et méritent d'être revisités au moyen d'une approche à la fois théorique et empirique, combinant démarche diachronique et comparative entre terrains diversifiés.

$\mathrm{Au}$ final, peu importent les termes pour désigner les terroirs, parcours ou communs maritimes; le plus important, ce sont les interactions, à savoir les institutions qui assurent la reproduction des liens forts entre les sociétés et la mer, et les processus de construction, déconstruction et réinvention de ces territoires.

\section{BIBLIOGRAPHIE}

Acheson J. 1975 - The lobster fiefs: economic and ecological effects of territoriality in the Maine lobster Industry. Human Ecology, 3(3) : 183-207.

Bennett N., Govan H. \& Satterfield T. 2015 - Ocean grabbing. Marine Policy 57 : 61-68.

Berkes F. 1989 - Common property resources: ecology and community-based sustainable development. London, Belhaven Press.

Berkes F., Feeny D., Mc Cay B. \& Acheson J.M. 1989 - The benefits of the commons. Nature 340 (13 july) : 91-93.

Blanc-Parmard C. \& Boutrais J. 1997 - Thème et variations : nouvelles recherches rurales au sud. Paris, ORSTOM, 367 p. (Colloques et séminaires). 
Bonnin M., Ly I., Queffelec B., Ngaido M. (Ed.) 2016 - Droit de l'environnement marin et côtier au Sénégal. Dakar, Sénégal, IRD, PRCM, 532 p.

Borrini-Feyerabend G., Pimbert M., Farvar M.T., Kothari A., Renard Y. 2009 - Partager le pouvoir : cogestion des ressources naturelles et gouvernance partagée de par le monde. Téhéran, IIUED et UICN/ CEESP/TGER, Cenesta, 499 p.

Braudel F. 1949 - La Méditerranée et le monde méditerranéen à l'époque de Philippe II. Paris, Armand Colin.

Brunet R., Ferras R. \& Théry H. 2005 [1992] - Les mots de la géographie. Dictionnaire critique. La Documentation Française Édition, (3e édition), 518 p. (Dynamiques du territoire).

Chauveau J.-P., Jul-Larsen E. \& Chaboud C. (Ed.) 2000 - Les pêches piroguières en Afrique de l'Ouest. Pouvoirs, mobilités, marchés. Paris, Karthala-CMI-IRD, 391 p.

Collet S. 1985 - Le tiers de l'espadon : un mode féodal d'appropriation de la ressource halieutique. Prémices pour une recherche sur la rente halieutique, Calabre. Anthropologie Maritime 2 : 41-53.

Compagnon D. \& Constantin F. 2000 - Administrer l'environnement en Afrique. Gestion communautaire, conservation et développement durable. Paris, Nairobi, Karthala - IFRA.

Corlay J.-P. 1979 - La notion d'espace de production halieutique : Proposition méthodologique d'étude à partir de l'exemple danois. Norois 104 : 449-466.

Cormier-Salem M.-C. 1992 - Gestion et évolution des espaces aquatiques : la Casamance. Paris, Orstom, 584 p. (Études et Thèses).

Cormier-Salem M.-C. 1995a - Paysans-pêcheurs du terroir et marins-pêcheurs du parcours. Les géographes et l'espace aquatique. L'espace géographique 1 : 46-59.

Cormier-Salem M.-C. 1995b - Terroirs aquatiques et territoires de pêche. Enjeux fonciers halieutiques des sociétés littorales ouest-africaines. In : Blanc-Pamard C., Cambrézy L. (Ed.), Terre, terroir, territoire. Les tensions foncières. Paris, Orstom : 57-82. (Colloques et Séminaires).

Cormier-Salem M.-C. 2000 - Appropriation des ressources, enjeu foncier et espace halieutique sur le littoral ouest-africain. In : Chauveau J.-P., Jul-Larsen E. \& Chaboud C. (Ed), Les pêches piroguières en Afrique de l'Ouest. Pouvoirs, mobilités, marchés. Paris, Karthala-CMI-IRD : 205-229.

Cormier-Salem M.-C. 2014 - Participatory governance of Marine Protected Areas: a political challenge, an ethical imperative, different trajectories. Senegal case studies. Sapiens 7 (2) 13 p. [En ligne] : http://sapiens.revues.org/1541

Cormier-Salem M.-C. 2015 - De la conservation à la concertation. Quelles AMP pour quelle gouvernance territoriale? In : Bonnin M., Laë R. \& Behnassi M. (Ed.), Les aires marines protégées ouest-africaines : défis scientifiques et enjeux sociétaux. Marseille, IRD : 97-116.

Cormier-Salem M.-C. 2017 - L'injonction du participatif dans la gouvernance des deltas ouestafricains : enjeux scientifiques, défis politiques. In : Cormier-Salem M.-C., Diakhaté M.M. \& Descroix L. (Ed.), Sciences participatives et gouvernance des patrimoines et territoires des deltas. Dakar, L'Harmattan, Actes du colloque PATEO/ PRCM : 9-30.

Cormier-Salem M.-C. \& Roussel B. 2002 - Patrimoines et savoirs naturalistes locaux. In : Martin J.Y. \& Leroy G. (Ed.), Développement durable? Doctrines, pratiques, évaluations. Paris, IRD : 125-142.

Cormier-Salem M.-C. \& Panfili J. 2016 - Mangrove reforestation in question: greening or grabbing coastal zones and deltas? African Journal of Aquatic Sciences 41 (1) : 89-98. 
Cormier-Salem M.-C. [sous presse] - Pêche en eau trouble : évolution des principes et outils de la gouvernance des littoraux ouest-africains. In : Croix K. de la \& Mitroi V. (Ed.), Écologie politique de la pêche. Paris (accepté pour publication PUF de Nanterre).

Cormier-Salem M.-C. [sous presse a] - An exploration of changes in mangrove tenure from a political ecology perspective. In : Artaud H. \& Surallés A. (Ed.) The sea within. Indigenous Territory and Perception of Marine Environment, IWTGA.

Cormier-Salem M.-C. [sous presse b] - Jeux d'échelles et solidarités territoriales. Vers une gouvernance multiscalaire des aires protégées. Paris, Journée d'études CEPED, 12 juin 2015. (accepté pour publication dans Quae).

Croix (de la) K. 2015 - Les territoires-temps des communautés de pêcheurs bozo et somono sur le Niger supérieur (Mali, Guinée). Thèse de doctorat en Géographie, Université Paris Ouest Nanterre La Défense, École doctorale Milieux, cultures et sociétés du passé et du présent.

Cury P. \& Cayré P. 2001 - Hunting became a secondary activity 2,000 years ago; marine fishing did the same in 2021. Fish and Fisheries (2) : 162-169.

Dahou T. \& Abdel Wedoud O.C. 2007 - L'autochtonie dans les Aires Marines Protégées. Terrain de conflits en Mauritanie et au Sénégal. Politique africaine 108 :173-190.

Demeritt D. 2015 - The promises of participation in science and political ecology. In : Perreault G.B.T. \& McCarthy J. (Ed.) The Routledge handbook of political ecology : 224-234.

Diouf P., Thiam D., Dia A., Ly M.E.M., Ndiaye N. A., Ngom F. \& Sané K. 1998 - Aménagement participatif des pêcheries artisanales du Sine-Saloum (Sénégal), Rapport final 2 de l'équipe pluridisciplinaire d'étude des écosystèmes côtiers du Sénégal. Programme environnement et développement dans les régions côtières et les îles, ISRA/CRODT.

Dugast S. 2002 - Modes d'appréhension de la nature et gestion patrimoniale du milieu. In : Cormier-Salem M.-C., Juhé-Beaulaton D., Boutrais J. \& Roussel B. (Ed.), Patrimonialiser la nature tropicale. Dynamiques locales, enjeux internationaux. Paris, IRD : 31-78. (Colloques et séminaires).

Fairhead J.L., Leach M \& Scoones M.I. 2013 - Green Grabbing. A new appropriation of Nature. London, Routledge.

Fall B. C. 2006 - La gestion des ressources naturelles dans l'organisation sociale et administrative traditionnelle des Lébous, un modèle de gouvernance traditionnel pour enrichir le débat sur le pluralisme juridique. [En ligne] : http://base.afrique-gouvernance.net/fr/corpus_dph/fiche-dph-741.html

Fay C. 1989 - Sacrifices, prix du sang, « eau du maître » : fondation des territoires de pêche dans le delta central du Niger (Mali). Cah. Sci. hum. 25 (1-2) : 159-176.

Gallais J. 1967 - Le Delta Intérieur du Niger, Études de géographie régionale. Paris, IFAN.

Gordon H.S. 1953 - An economic approach to the optimum utilization of fisheries resources. Journal of the Fisheries Research Board of Canada 10 : 442-457.

Gordon H.S. 1954 - The economic theory of a common property resource: the fishery. Journal of Political Economy (62) : 124-142.

Granier L. 2006 - Les conventions locales de gestion des ressources naturelles et de l'environnement. Légalité et cohérence en droit sénégalais. Gland, Suisse, UICN, Cambridge, Royaume-Uni, $\mathrm{x}+44$ p.

Hardin G. 1968 - The tragedy of the commons. Science $162: 1243-1248$.

Lavigne Delville P. 2001 - Quelles gouvernance pour les ressources renouvelables dans le contexte de la décentralisation en Afrique de l'Ouest. Études de l'AFD (Agence française de Développement). 
MacCay B. \& Acheson J.M. (Ed.) 1987 - The question of the commons: the culture and ecology of communal resources. Tucson, Arizona, University of Arizona Press, $440 \mathrm{p}$.

Mathevet R., Thompson J.D., Delanoë O., Cheylan M., Gil-Fourrier C. \& Bonnin M. 2010 - La solidarité écologique : un nouveau concept pour la gestion intégrée des parcs nationaux et des territoires. Natures Science Sociétés $18:$ 424-433.

Mbaye A. 2013 - Système de gestion des ressources et pluralité des règles. In : Fontana A. \& et Samba A. (Ed.), Artisans de la mer : une histoire de la pêche maritime sénégalaise. Dakar, Sénégal, WWF-PNUD- FNRAA-UE-CRODT-Cofrepeche : 90-96.

Mbaye A., Thiao D., Diadhiou H. D. \& Dème M. 2013 - Diagnostic participatif de la Gouvernance des aires marines protégées au Sénégal. Dakar, ISRA/CRODT, Rapport d'activité, 41 p.

Mbaye A. \& Cormier-Salem M.-C. 2015 - La gouvernance des pêcheries en Casamance : entre les aires protégées des autochtones, le repos biologique des crevettes et la fermeture de la pêche par l'administration. In : Descroix L., Djiba S., Sané T. \& Tarchiani V. (Ed.) Eaux et sociétés face au changement climatique dans le bassin de la Casamance. Paris, L'Harmattan : 169-187.

Mbaye A., Cormier-Salem M.-C. \& Fall A. S. 2016 - Delta du Saloum : nouvelle gouvernance des ressources à travers un retour aux savoirs empiriques. Communication présentée au Colloque international PATEO : Sciences participatives et gouvernance des patrimoines et territoires des deltas, UGB, Saint-Louis, Sénégal, 11-13 mai 2016.

Mbaye A, Thiam N. \& Fall M. 2018 - Les zones de pêche protégées au Sénégal : entre terroir du pêcheur et territoire du poisson. Quelle(s) échelle(s) de gestion? Développement durable et territoires - Économie, géographie, politique, droit, sociologie 9 (1).

Ostrom E. 1990 - Governing the Commons: the Evolution of Institutions for Collective Action. Cambridge, Cambridge University Press.

Ostrom E., Dietz T., Dolsak N., Stern P.C., Stonich S. \& Weber E.U. (Ed.) 2002 - The drama of the commons. Washington D.C., National Academy Press, 435 p.

Pauly D. 1998 - Overfishing Disrupts Entire Ecosystems. Science Now February 5.

Pélissier P. 1995 - Transition foncière en Afrique noire. Du temps des terroirs au temps des finages. In : Blanc-Pamard C. \& Cambrézy L. (Ed.) Terre, terroir, territoire. Les tensions foncières. Paris, Orstom : 19-34. (Colloques et Séminaires).

Pélissier P. \& Sautter G. 1970 - Bilan et perspectives d'une recherche sur les terroirs africains et malgaches (1962-1969). Études Rurales 37-38-39 : 7-46.

Péluso N.L. 1993 - Coercing Conservation? The Politics of State Resource Control. Global Environmental Change 3 (20) :199-216.

Péluso N. \& Watts M. 2001 - Violent Environments. Ithaca, Cornell University press.

Perreault T., Bridge G. \& McCarthy J. (Ed.) 2015 - The Routledge handbook of political ecology. Abingdon, Oxon ; New York, NY, Routledge, 646 p.

Pliya J. 1985 - Protection du milieu et législation traditionnelle de protection en Afrique Ouest. Communication au colloque de Cotonou, déc., $10 \mathrm{p}$.

Revéret J.-P. 1991 - La pratique des pêches. Comment gérer une ressource renouvelable. Paris, Harmattan, 198 p. (Environnement).

Ribot J., Agrawal A. \& Larson A. M. 2006 - Recentralizing While Decentralizing: How National Governments Reappropriate Forest Resources. World Development $11: 1864-1886$. 
Ruddle K. \& Akimichi T. 1984 - Maritime institutions in the Western Pacific. Senri Ethnological Studies $17: 333$.

Ruddle K., Hviding E. \& Johannes R.E. 1992 - Marine resources management in the context of customary tenure. Marine Resource Economics 7 : 249-273.

Sarr O., Queffelec B., Cormier-Salem M.-C. \& Boncoeur J. 2016 - Labellisation of products as a mechanism for environmental justice. Case study of dried shellfish in Saloum Delta Biosphere Reserve (Senegal). AMURE publications, Working Paper series D 39-2016, 21 p. [En ligne] : http:// www.umr-amure.fr/electro_doc_amure/D_39_2016.pdf

Saturnino M.B. Jr., Hall R., Scoones I., White B. \& Wolford W 2011 - Towards a better understanding of global land grabbing: an editorial introduction. JPeasantStud 38 (2) : 209-216.

Sautter G. 1973 - Recherches en cours sur les villes d'Afrique Noire. Thèmes et problèmes. Point de vue d'un géographe. Cahiers d'Études Africaines 13 (51) : 405-416.

Sautter G. \& Pélissier P. 1964 - Pour un atlas des terroirs africains. Structure type d'une étude de terroir. L'Homme, janv.-avril 1964, IV, 56-72.

Schlosberg D. 2013 - Theorising environmental justice: the expanding sphere of a discourse. Environmental Politics $22: 37-55$.

Sikor T. \& Newell P. 2014 - Globalizing environmental justice? Geoforum 54 :151-157.

Verdeaux F. 1992 - Société de pêcheurs et environnement : savoirs et appropriation halieutique du milieu. Afrique Contemporaine 61 (spécial), L'environnement en Afrique :125-144.

Walker G. 2012 - Environmental justice: concepts, evidence and politics. London, Routledge.

Zimmerer K. 2006 - Globalization and new geographers of conservation. Chicago, University of Chicago press, $357 \mathrm{p}$.

Zimmerer K. \& Bassett T. J. (Ed.) 2003 - Political ecology. An integrative approach to geography and environment-development studies. New-York, The Guilford press, $310 \mathrm{p}$.

\section{NOTES}

1. Dans un article, Cormier-Salem (sous presse a), intitulé Pêche en eau trouble: évolution des principes et outils de la gouvernance des littoraux ouest-africains), nous analysons en détail les changements de paradigmes qui inspirent ces politiques, de la «gestion rationnelle des ressources halieutiques » qui prédominent jusqu'aux années 1970 à la "gouvernance durable et partagée des territoires et des patrimoines » qui tend à s'imposer à partir des années 1990.

2. L'analyse de l'impact des politiques environnementales sur les dynamiques locales est au cœur des réflexions de l'UMR Paloc «Patrimoines locaux et gouvernance» (www.paloc.fr) et du Laboratoire Mixte International PATEO « Patrimoines et territoires de l'eau » (www.pateo.ird.fr), dans lesquels s'inscrivent nos travaux.

3. Pour ne pas alourdir le texte, nous n'utiliserons que le déterminant « maritime », en le prenant au sens large (du littoral- estran, plage, trait de côte- à la haute mer)

4. La justice environnementale est examinée dans ces trois dimensions (Schlosberg 2013, Walker 2012, Sikor \& Newell 2014) : la distribution et l'allocation des ressources halieutiques (régulation, partage des bénéfices directs et indirects issus de la conservation de la biodiversité marine, tenures maritimes, etc.) ; les procédures relatives aux décisions (qui participe à la gouvernance des espaces maritimes? qui décide? quels sont la place et le rôle des groupes locaux, et notamment des pêcheurs artisans?); la reconnaissance des cultures, savoirs et besoins des 
différents groupes dans ces processus, et notamment l'institutionnalisation des terroirs maritimes.

5. Ces processus peuvent être publics (domaine public, ZEE, AMP, etc.), privés (grands barrages, fermes aquacoles, etc.), ou encore hybrides comme dans le cas du reboisement des mangroves, qui, dans le cadre de REDD+, implique bien souvent des institutions publiques, des ONGs et des entreprises privées (Cormier-Salem \& Panfili 2016). Dans cet article, il sera surtout question d'enclosure publique, quoique la privatisation des parcs nationaux soit une tendance majeure en Afrique (comme celui du delta du Saloum).

6. Nous disons bien «entre autres » car il ne faudrait pas faire des pêcheurs des écologistes qui s'ignorent. Comme l'exprime très bien S. Dugast (2002), ce qui préside à l'élaboration de ces règles, c'est la reproduction sociale. Les visées écologistes, explicites ou implicites (Ruddle et al. 1992) sont secondaires.

\section{RÉSUMÉS}

La diversité des formes de socialisation de l'espace maritime et de spatialisation des sociétés maritimes se traduit dans la richesse des termes pour désigner les droits traditionnels d'appropriation de la mer et de ses ressources, des TUR'f (Territorial Use Rights in fisheries) au CMT (Customary Marine Tenure), des terroirs halieutiques aux technotopes.

Cet article se propose de revisiter la notion de terroir maritime, en s'appuyant sur des études empiriques menées en particulier au Sénégal dans les années 1980 et une analyse de la littérature internationale en sciences sociales et en ré-explorant cette notion à partir de terrains diversifiés, du Sénégal au Vietnam et à l'aune des discours et dispositifs environnementaux récents.

Cette notion, typiquement francophone, voire Africaniste, utilisée à l'origine pour désigner les portions de territoire dont dépendent les communautés rurales pour leur subsistance, nous avait semblée pertinente pour qualifier les espaces littoraux et maritimes contrôlés par les paysanspêcheurs et que nous distinguions des parcours des marins-pêcheurs. Dès l'époque coloniale puis avec les Indépendances, ces espaces, non aménagés de façon permanente, appropriés bien souvent collectivement (ce sont des «commons» ou communs), font l'objet d'accaparement public et privé

Dans cet article, nous discutons de la pertinence de cette notion de terroir maritime et proposons un cadre conceptuel et méthodologique. Nous retraçons la chronique de la fin de ces terroirs ou de leur remise en cause avec les processus d'enclosure publique et privée (domaine public, ZEE, AMP, etc.); puis nous analysons leur renouveau, compte tenu du changement de paradigme en termes de conservation de la biodiversité et des nouvelles approches de cogestion (ou gouvernance partagée et durable) se focalisant sur le local. Cette dynamique, est discutée à travers divers exemples ouest-africains, de l'échelle internationale à l'échelle locale: le projet de territorialisation des espaces maritimes ouest-africains de la Banque Mondiale, l'acte III de la décentralisation, achevant le processus de communalisation des terroirs traditionnels et la création des aires ou zones marines protégées au Sénégal, dites communautaires ou patrimoniales telles les APAC et les ZPP, ou encore l'engouement actuel pour les produits de terroir ou de meroir.

The diverse forms of sea spaces' socialization and coastal societies'spatialization is reflected in the wealth of words for designating the traditional property rights of the sea and its resources. 
This paper intends to revisit the notion of marine "terroir", based on empirical studies, particularly in Senegal in the 1980s and an analysis of the international literature in the social sciences, and re-exploring the concept from diverse fieldworks, in West Africa and in the light of recent discources and mecanisms regarding the sea policies.

This concept of terroir, typically French and even Africanist, was originally used to designate portions of territory which rural communities depend for their livelihoods. It seems relevant to qualify the coastal and maritime areas controlled by the farmers-fishers and that we distinguish from the moving territories of fishers. From colonial times and then with the Independence, these spaces, not permanently managed, but owned and controlled collectively (they are "commons"), are subject to public and private grabbing.

In this paper, we discuss the relevance of this concept of sea terroir and propose a conceptual and methodological framework. We trace the chronicle of the end of the traditional tenure and their questioning with public and private enclosure process (public domain, EEZ, MPA, etc.); then we analyze their revival, given the changes of paradigm in terms of biodiversity conservation and the new approaches to co-management (or shared and sustainable governance), focusing on the local level. This dynamic is discussed through various West African examples, from global to local scales: the proposed regionalization of West African sea spaces of the World Bank, Act III of decentralization, completing the process of communalization of traditional terroirs and the creation of Marine Protected Areas and Areas of Community Heritage or the current craze for local sea-food products or meroir.

\section{INDEX}

Keywords : coastal and sea spaces, commons, tragedy, territory, regulation, actors, politics, fishery, local knowledge, institutional innovation

Mots-clés : espaces littoraux et maritimes, communaux, tragédie, territoire, régulation, pêche, État, acteurs, savoirs locaux, innovation institutionnelle

\section{AUTEURS}

\section{MARIE-CHRISTINE CORMIER-SALEM}

DR IRD en sciences sociales (géographie, écologie politique), UMR PALOC IRD/MNHN, Sorbonne Université, CP 026, 57 rue Cuvier 75231 Paris cedex 05

\section{ADAMA MBAYE}

Doctorant MNHN, Anthropologie, UMR PALOC/ISRA/CRODT BP 1386 Dakar, Sénégal 